(C) 1999 IEEE. Reprinted from IEEE Transactions on Biomedical Engineering, 46(12).

This material is posted here with permission of the IEEE. Such permission of the IEEE does not in any way imply IEEE endorsement of any of The University of Melbourne's products or services. Internal or personal use of this material is permitted. However, permission to reprint/republish this material for advertising or promotional purposes or for creating new collective works for resale or redistribution must be obtained from the IEEE by writing to pubs-permissions@ieee.org.

By choosing to view this document, you agree to all provisions of the copyright laws protecting it. 


\title{
The Effects of Stochastic Neural Activity in a Model Predicting Intensity Perception with Cochlear Implants: Low-Rate Stimulation
}

\author{
Ian C. Bruce,* Member, IEEE, Mark W. White, Member, IEEE, Laurence S. Irlicht, Member, IEEE, \\ Stephen J. O'Leary, and Graeme M. Clark
}

\begin{abstract}
Most models of auditory nerve response to electrical stimulation are deterministic, despite significant physiological evidence for stochastic activity. Furthermore, psychophysical models and analyses of physiological data using deterministic descriptions do not accurately predict many psychophysical phenomena. In this paper we investigate whether inclusion of stochastic activity in neural models improves such predictions. To avoid the complication of interpulse interactions and to enable the use of a simpler and faster auditory nerve model we restrict our investigation to single pulses and low-rate $(<200$ pulses/s) pulse trains. We apply signal detection theory to produce direct predictions of behavioral threshold, dynamic range and intensity difference limen. Specifically, we investigate threshold versus pulse duration (the strength-duration characteristics), threshold and uncomfortable loudness (and the corresponding dynamic range) versus phase duration, the effects of electrode configuration on dynamic range and on strength-duration, threshold versus number of pulses (the temporal-integration characteristics), intensity difference limen as a function of loudness, and the effects of neural survival on these measures. For all psychophysical measures investigated, the inclusion of stochastic activity in the auditory nerve model was found to produce more accurate predictions.
\end{abstract}

Index Terms - Auditory nerve, cochlear implant, dynamic range, functional electrical stimulation, intensity difference limen (IDL), psychophysics, stochastic model, threshold, uncomfortable loudness.

\section{INTRODUCTION}

$\mathbf{M}$ ODELS OF cochlear implant perception have historically utilized deterministic descriptions of auditory nerve (AN) response to electrical stimulation. Recently, physiological models of AN response have been developed that

Manuscript received February 18, 1998; revised June 7, 1999. This work was supported by the Human Communication Research Centre, The University of Melbourne, the Bionic Ear Institute, and the CRC for Cochlear Implants, Speech and Hearing Research. Asterisk indicates corresponding author.

*I. C. Bruce was with the Bionic Ear Institute, the Department of Otolaryngology, The University of Melbourne, East Melbourne VIC 3002, Australia, and is now with the Department of Biomedical Engineering, 505 Traylor Building, Johns Hopkins University, 720 Rutland Ave., Baltimore, MD 21205 USA (e-mail: ibruce@bme.jhu.edu).

M. W. White is with the Department of Electrical and Computer Engineering, North Carolina State University, Raleigh, NC 27695 USA.

L. S. Irlicht was with the Bionic Ear Institute and the Department of Otolaryngology, The University of Melbourne, East Melbourne VIC 3002, Australia, and is now with County Investment Management, Melbourne VIC 3000, Australia.

S. J. O'Leary and G. M. Clark are with the Bionic Ear Institute and the Department of Otolaryngology, The University of Melbourne, East Melbourne VIC 3002, Australia.

Publisher Item Identifier S 0018-9294(99)09302-7. incorporate stochastic activity [1]-[9], but the consequences for behavioral performance have not been investigated.

In the normal mammalian auditory system, the AN exhibits both spontaneous discharges and variance in acousticallydriven discharge rates. The inner-hair-cell/auditory-nerve synapse appears to be the primary source of this stochastic activity [10]. In most cases, profound sensorineural hearing loss results from the functional loss of all, or nearly all, hair cells. As a consequence, hair cells are unlikely to be a significant source of variance (i.e., noise) in neural response to electrical stimulation of a profoundly deaf ear [11]. A series of studies by Verveen and colleagues [12] have shown, however, that random fluctuations in a neuron's membrane potential produce a secondary source of noise. The magnitude of these fluctuations is significantly less than the noise from the inner hair cells and has consequently been regarded as unimportant. Hence, neural response to electrical stimulation has historically been modeled via deterministic descriptions such as the Hodgkin-Huxley equations. However, significant variance has been measured in the response of AN fibers to single biphasic current pulses [1], [5], [13] and pulse trains [2], [3] that cannot be explained at all by deterministic models. This stochastic activity should be a significant factor in the psychophysical performance of cochlear implant users.

Studies of single fiber response using an arbitrary measure of AN fiber threshold do not accurately predict behavioral threshold versus phase duration (strength-duration) curves for sinusoidal stimulation [14] or for pulsatile stimulation [15], [16]. Indeed, strength-duration curves of cochlear implant users are not well predicted by deterministic models of AN response to electrical stimulation [16], [17].

In this paper we investigate whether these and other inaccuracies in predictions of intensity perception could be due to ignoring the stochastic response of the AN to electrical stimulation. In order to avoid the complication of interpulse interactions and to enable the use of a simpler and computationally faster AN model, we restrict our investigation in this paper to single biphasic pulses and low-rate $(<200 \mathrm{pps})$ pulse trains. We derive a model of intensity perception based on the model of neural excitation developed in [1] and compare model predictions for the deterministic and stochastic descriptions of AN response to electrical stimulation. We develop the psychophysical section of the model in such a way that signal detection theory can be applied to predict 


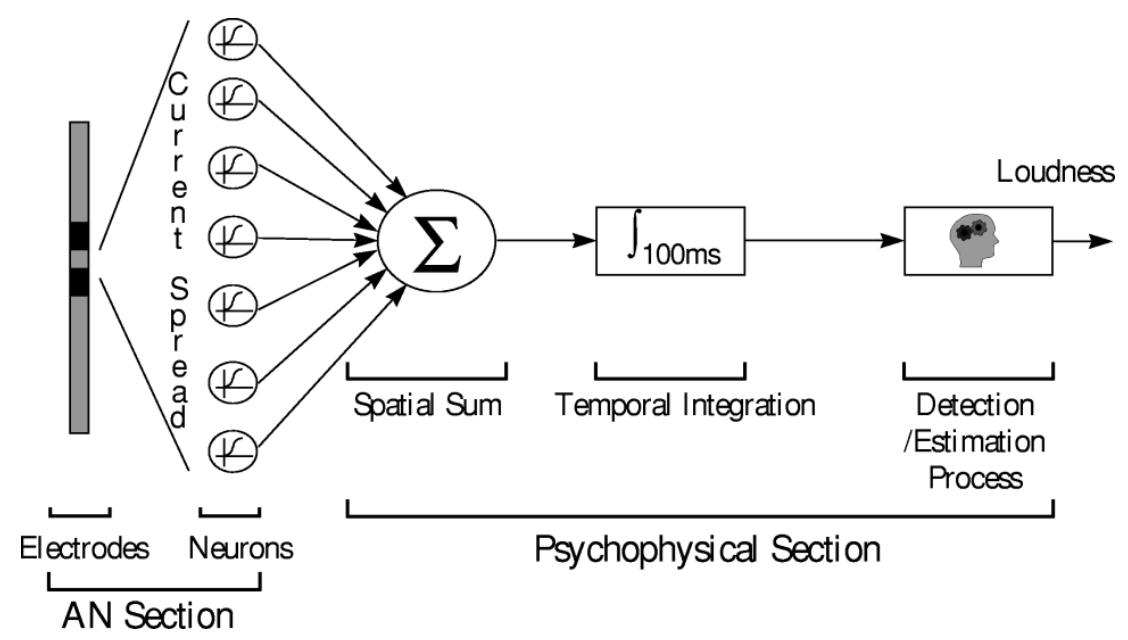

Fig. 1. Cochlear neural model and behavioral intensity-discrimination/loudness model.

directly how behavioral threshold, dynamic range and intensity difference limen (IDL) change with stimulus parameters and nerve survival.

\section{METHODS}

\section{A. Psychophysical Model}

The form of our psychophysical model is given in Fig. 1. This model follows the approach of [18]-[21], in which intensity perception is related to the response of the AN to an electrical stimulus. Such models can be derived from computational psychophysical models of acoustic stimulation that relate intensity perception to AN response (e.g., [22]-[25]). Here, we assume that the peripheral section of acoustic psychophysical models can be attributed to excitation of the AN and the remaining components attributed to more central processing. To construct the model, the excitation section is replaced by a model of electrical stimulation of the AN. The central section of the model is similar to the central section used to model normal hearing. Accordingly, our model has two major divisions, an auditory nerve section and a psychophysical section.

1) AN Section: In this paper, two models of the AN are compared: a stochastic model and a deterministic model. For the AN section of our model we utilize a description of total AN response to electrical stimulation based on the model of White [7], [9] that we developed further in [1]. This model consists of: 1) an input/output (I/O) function for each of an arbitrary number of AN fibers and 2) a function approximating the attenuation of the excitatory current as it spreads from the active electrode to the site of action potential generation in each neuron.

The I/O function describes the probability of discharge in response to a single pulse as a function of the stimulus intensity delivered to the fiber (expressed in absolute units). For the deterministic model, this is a step function [1, Eq. 2], where the intensity at which the discharge probability changes from zero to one is referred to as the fiber's threshold. For the stochastic model, the I/O function is an integrated-Gaussian [1, Eq. 3], where the intensity corresponding to a discharge probability of 0.5 is referred to as the fiber's threshold. The slope of the integrated-Gaussian is determined by the relative spread (RS), which is the standard deviation of the Gaussian noise divided by the threshold [1, Eq. 4]. The Gaussian noise is the underlying mechanism that generates the integrated-Gaussian I/O function.

Although only minimal physiological data have been collected on the distributions of thresholds and RS's in a population of AN fibers at any cochlear location, there exist just enough to enable us to set distributions of model parameters to approximate those seen in the physiological data from cat [1]. We model the threshold and RS of each fiber as two independent random variables. Pseudorandom numbers are generated using the estimated distributions to obtain different thresholds and different RS's for each fiber. In order to maintain the same set of I/O functions across simulations with the same number of fibers, the seeds of the pseudorandom number generators are two different fixed values, one for the generation of the thresholds and one for the RS's.

For both the deterministic and the stochastic model, fiber thresholds are uniformly distributed from $-5 \mathrm{~dB}$ to $+5 \mathrm{~dB}$ with respect to the mean threshold, which is determined by

$$
\begin{aligned}
E[\text { Threshold }] & =\text { Mean Threshold } \\
& =121.04 \times \mathrm{PW}^{-0.18}(\mathrm{~dB} \text { re. } 1 \mu \mathrm{A})
\end{aligned}
$$

where PW is the pulse width (phase duration) in $\mu \mathrm{s} / \mathrm{phase}$. Fiber thresholds are uniformly distributed in units of $\mathrm{dB}$ re. $1 \mu \mathrm{A}$.

For the stochastic model, the RS's are normally distributed, with the mean determined by

$\mathrm{E}[\mathrm{RS}]=0.12+9.51 \times 10^{-5} \times \mathrm{PW}-7.90 \times 10^{-9} \times \mathrm{PW}^{2}$

and a standard deviation of 0.06 .

An integrated-Gaussian function describes a fiber's probability of discharge versus absolute intensity, not on a relative (e.g., dB) scale. For each fiber, threshold is expressed in absolute units and the standard deviation of the Gaussian noise is the fiber's RS multiplied by its threshold expressed in absolute units. 
Following [18], we approximate the electrode array by a point source of current at the active electrode and the AN tissue by a homogeneous resistive medium consisting of a uniform density of single AN fibers. Unless otherwise noted, the model cochlea is populated with 10000 fibers. We assume that the stimulating electrode is placed $15 \mathrm{~mm}$ inside a $30 \mathrm{~mm}$ cochlea. To calculate the stimulus intensity at each AN fiber, we assume that the stimulus is attenuated at the rate of $0.5 \mathrm{~dB} / \mathrm{mm}$ for monopolar (MP) stimulation [26] and $4 \mathrm{~dB} / \mathrm{mm}$ for bipolar (BP) stimulation-the latter value is appropriate for both radial-BP pairs [26] and closely-spaced longitudinalBP pairs [27]. Less closely-spaced BP configurations should produce a lower rate of attenuation, such that model responses and the resulting predictions will tend toward those given for MP configurations.

Note that in order to simplify model-data comparisons, we ignore such factors as phase order for biphasic stimuli (anodic/cathodic or cathodic/anodic) and electrode form (shape and material). Future investigation of such factors may suggest modifications to the model of [1] that would allow their inclusion in predictions of psychophysical data.

2) Psychophysical Section: Following [18] and [21], intensity discrimination is based on the total discharge rate of all AN fibers activated by the electrical stimulation. Following [21], from psychophysical measurement of threshold versus stimulus duration [28], [29], we use a 100-ms rectangular integration window. ${ }^{1}$ These two aspects, the summing of responses across all neurons and the long-term temporal integration, can be modeled by spatial summation and temporal integration of the output of the neural section.

Note that if our model were to be extended to investigate psychophysical results for higher pulse-rate stimuli, short-term integration effects could be included in the psychophysical section using the approach of McKay and McDermott [19] or included in the AN section [5], [29], [30]. Such short-term integration effects may be particularly apparent in amplitude modulated pulse trains [31]. Note also that for higher pulse rates, in order to take into consideration refractory effects, the pulse-train model of [2] should be used in the AN section of the model in place of the single-pulse model of [1] used in this paper. See [2] for a discussion of further interpulse effects that may need to be considered.

\section{B. Determination of Psychophysical Measures}

Signal detection theory refers to statistical methods for determining when a signal may be detected in the presence of some confounding signal (e.g., background noise). The signal to be detected and the confounding signal may also be considered as a pair of signals to be distinguished, and thus such methods will equally apply to signal discrimination tasks. Statistical methods are required when either one or both of the

\footnotetext{
${ }^{1}$ If the stimulus is $<100 \mathrm{~ms}$, as is the case for almost all the data presented in this paper, the integrator's output is simply the sum of all spikes that occur during the 100-ms integration period. For cases where the stimulus is $>100$-ms, we sum the responses elicited by the maximum number of pulses that can appear within the temporal integration window. For a uniform pulse train this is equal to the elicited response to $n$ pulses, where $n$ is the pulse rate times $100 \mathrm{~ms}$.
}

signals contain some variability. Signal detection theory has formed the basis of many successful psychophysical models of the normal auditory system (e.g., [22]-[25]). Indeed, signal detection theory is the basis for models of many sensory systems. We believe that signal detection theory will also be required in electrical stimulation of the auditory nerve where stochastic activity results from membrane noise. Although statistical methods are unnecessary for the deterministic model, we consider it as a specialized case of the stochastic model where the variances are equal to zero.

The output of the neural/psychophysical model developed in Section II-A can be used to predict psychophysical results using either Monte Carlo or analytical signal detection theory methods. Monte Carlo techniques are useful because they can incorporate the same paradigm with which psychophysical results are collected. They are also more accurate because they can incorporate the actual measured response distribution. Analytical methods on the other hand are more efficient computationally, but require approximation of the response distribution.

In the following two sections we describe the methods used in determining our model's performance on a number of different psychophysical tasks.

1) Threshold and Intensity Difference Limen: In the Monte Carlo implementation of the model, a pseudorandom uniformly-distributed number between zero and one is generated for each neuron. If the number is less than the probability of discharge for that neuron [as determined by the model neuron's I/O function and the stimulus parameters], the neuron is deemed to have discharged. This is repeated for each pulse in the pulse train. The total number of discharges for all neurons to each pulse is then passed through the psychophysical section of the model to give the total number of discharges occurring within the temporal integration period. This output can then be used directly in any criterion-specific psychophysical paradigm.

A range of psychophysical paradigms were used in the collection of the behavioral thresholds and IDL presented in Section III, some of which were not criterion-specific. For consistency and simplicity, we use a standard two-interval forced-choice paradigm [32] to determine the model's prediction of the psychophysical data. From Section II-A, two stimuli are perceived to be equal in intensity when the output of the temporal integration section of the model is identical for the two stimuli. If we assume an ideal observer, then the stimulus interval eliciting the greater number of discharges is chosen as the one containing the stimulus, in the case of a threshold measurement. In the case of an IDL measurement, the interval with the larger spike-count is chosen as the interval containing the higher intensity stimulus. In the rare case when the number of discharges is equal for the two intervals an "unbiased coin is flipped" to generate the decision.

In analytical applications of the model, the distribution of the model's output is approximated by an analytical function. Then signal detection theory is used to derive an analytical expression to predict the model's performance on any criterionspecific psychophysical detection/discrimination task. Next we 
derive expressions for predicting the model's performance on a two-interval forced-choice paradigm.

Consider two discrete random variables $X_{1}$ and $X_{2}$ that describe the number of discharges, as predicted by the model developed in Section II-A, produced in response to stimuli one and two, respectively. $X_{1}$ and $X_{2}$ have probability mass functions $f_{1}$ and $f_{2}$ with means of $\mu_{1}$ and $\mu_{2}$ and standard deviations of $\sigma_{1}$ and $\sigma_{2}$, respectively. $f_{1}$ and $f_{2}$ have values for all integers within the bounds $\left[0, X_{\max }\right]$, where $X_{\max }$ is the maximum number of discharges possible.

If stimulus two has the greater intensity, the probability of our detection/discrimination system choosing correctly that it has a greater intensity than stimulus one is

$$
\begin{aligned}
\operatorname{Pr}\{\text { Choosing correctly }\}= & \sum_{n=0}^{X_{\max }}\left(f_{1}(n) \sum_{m=n+1}^{X_{\max }} f_{2}(m)\right) \\
& +\frac{1}{2} \sum_{n=0} X_{\max } f_{1}(n) f_{2}(n) .
\end{aligned}
$$

Proof: See [33, p. 168]. From [1], if $\mu_{i}<15$ and $X_{\max } \geq 100, f_{i}$ is well approximated by the Poisson probability mass function

$$
f_{i}\left(x ; \mu_{i}\right)=\frac{e^{-\mu_{i}} \mu_{i}^{x}}{x !} \quad x=0,1,2, \ldots
$$

and if $15 \leq \mu_{i} \leq X_{\max }-15$ and $X_{\max } \gg 30, f_{i}$ is well approximated by the Gaussian probability density function

$$
f_{i}\left(x ; \mu_{i}, \sigma_{i}\right)=\frac{1}{\sqrt{2 \pi} \sigma_{i}} e^{-\left(x-\mu_{i}\right)^{2} / 2 \sigma_{i}^{2}}-\infty<x<\infty .
$$

Equation (3) assumes both $X_{1}$ and $X_{2}$ are discrete random variables having integer values bound by $\left[0, X_{\max }\right]$. There are two possible methods of using (5) for the case where $\mu_{i} \geq 15$. Either the appropriate sums in (3) can be changed to integrals, or the Gaussian probability density function can be discretized such that it only has values for integers within the bounds $\left[0, X_{\max }\right]$, forming a probability mass function with the same formula as (5). These methods produce virtually identical results. We use the latter method because it is computationally faster.

The derivation of (3) follows the traditional methods used in analytical signal detection theory (e.g., see [22]) except in two manners: 1) while unequal variance may have been traditionally allowed for in signal detection noise models, it is not usual to allow for two different noise distributions as we have done and 2) continuous random variables are normally used, rather than discrete random variables as we have done. Both of these deviations are necessitated and justified by what is known from the neurophysiology [1].

Sample psychometric functions for behavioral threshold generated using this method are plotted in Fig. 2. These were obtained from the model of 10000 neurons $^{2}$ in response to a single $100-\mu \mathrm{s} /$ phase biphasic pulse generated with a MP electrode configuration. The deterministic model produces a step-shaped psychometric function, because there is no variance in the number of spikes. With the ideal observer assumed

\footnotetext{
${ }^{2}$ This is a conservative estimate of the number of surviving AN fibers in a subject with a profound sensorineural hearing loss [34].
}

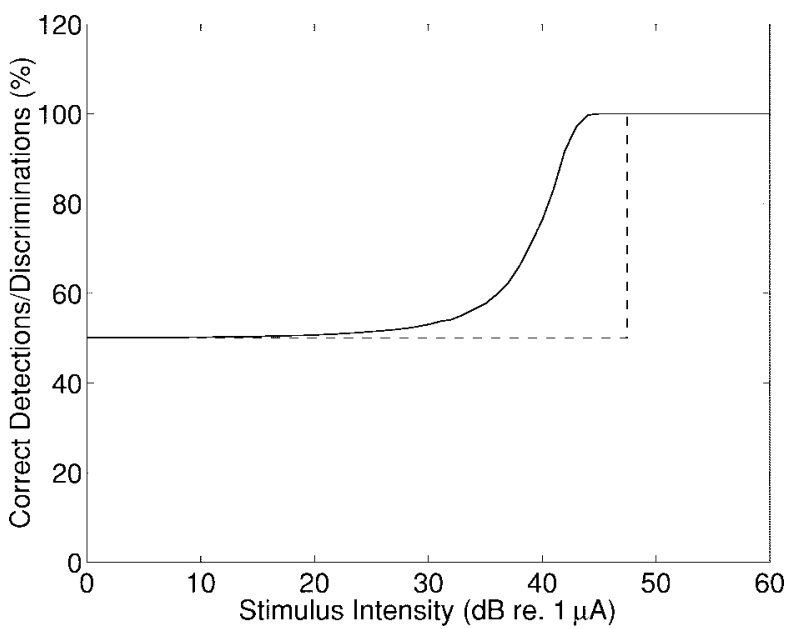

Fig. 2. Psychometric functions for behavioral threshold predicted by the deterministic (dashed line) and stochastic (solid line) AN models.

in our model, a discriminable difference for the deterministic model corresponds to the lowest intensity difference producing an increase in the spike count. In contrast, the stochastic model produces a psychometric function that rises smoothly from 50\% (chance performance level) to $100 \%$ discrimination as the neural responses become more discriminable, i.e., as the overlap of the response distributions of the two stimulus conditions decreases.

A point on the psychometric function that is defined as the threshold (or IDL) can then be chosen to match the paradigm that was used in the collection of the psychophysical data that we wish to predict. However, not all of experimental methods used to collect the data presented in the results were criterionspecific. In such cases we used $70.71 \%$ as the criterion. ${ }^{3}$ For a $70.71 \%$ criterion, a discriminable difference for the stochastic model corresponds to the intensity at which $70.71 \%$ of the time more spikes are produced by the comparison interval than by the reference interval.

In order to test the accuracy of the analytical estimate, we conducted Monte Carlo simulations of a standard up-down threshold procedure [32] using our psychophysical model. For each threshold measurement the simulation was run until ten turning points were reached. Threshold was taken to be the mean of the final eight turning points. The points (o) plotted in Fig. 3 are the means of four of these threshold measurements for four different phase durations and three different durations (given in number of pulses) of a 100 pps pulse-train, with error bars indicating \pm 1 standard deviation. Thresholds obtained via the analytical method (lines) accurately estimate the simulation results.

2) Dynamic Range: We define dynamic range as the difference in $\mathrm{dB}$ between behavioral threshold and the lowest stimulus level to elicit an uncomfortably loud percept. One longstanding and intensively studied hypothesis is that loudness is simply proportional to the total AN response (e.g., [35]). While there is now direct evidence to suggest that loudness

\footnotetext{
${ }^{3}$ The point on a psychometric function that is estimated by a two-down, one-up procedure is $70.71 \%$ [32]. We performed sensitivity testing and found that comparisons between the deterministic and the stochastic model were relatively insensitive to the value of the criterion.
} 


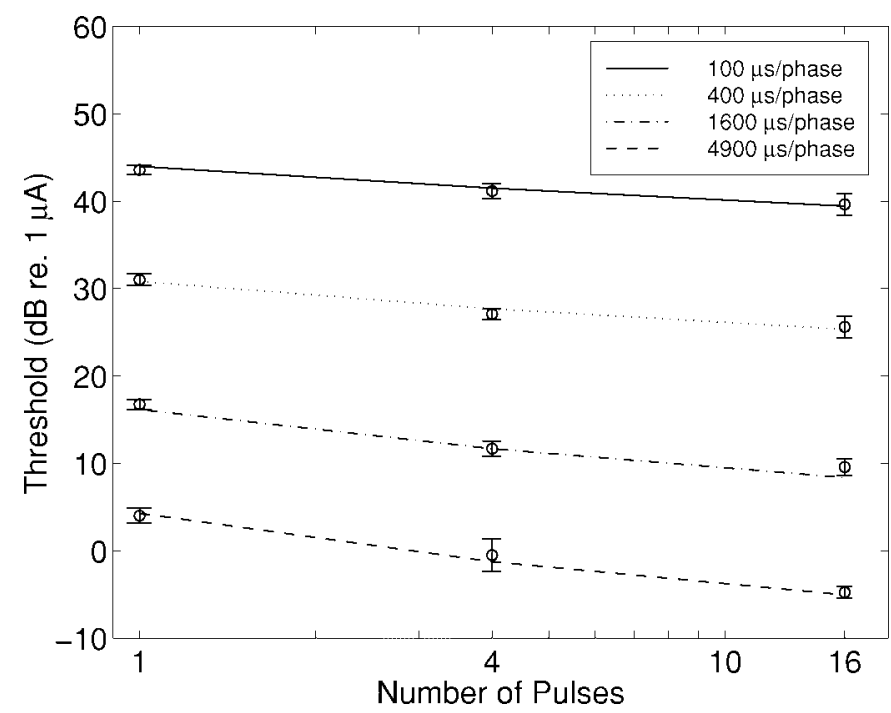

Fig. 3. Mean thresholds from four iterations of Monte Carlo simulations (o), with error bars indicating \pm 1 standard deviation, compared with thresholds obtained via the analytical method (lines).

is not directly proportional to the total AN response, loudness does appear to be monotonically related to the neural response [36]. Therefore, we assume here that uncomfortable loudness (UCL) corresponds to a fixed number of neural discharges for the whole AN within the period of temporal integration, i.e., the output of the model's temporal integrator section. We will call the number of discharges required to reach uncomfortable loudness $N_{u c l}$. We have no plausible way of determining how many responses correspond to UCL, ${ }^{4}$ so we, therefore, present predictions for three different values of $N_{u c l}$, which appear to best account for the psychophysical data.

\section{RESULTS}

Here we present: 1) the model's response properties as a function of stimulus and model parameters and 2) some examples of the model's prediction of psychophysical data.

\section{A. Model Response Properties}

Model response versus stimulus intensity predicted by our model with 10000 fibers in response to a single pulse of duration 100 and $2000 \mu \mathrm{s} /$ phase is plotted in Fig. 4. The number of discharges is a monotonic function of the stimulus intensity, and there is horizontal shift of the function dependent on the phase duration.

Plotted with a linear ordinate [Fig. 4(a)], the deterministic model and the stochastic model appear to predict very similar mean response growth curves. However, when plotted with a logarithmic ordinate [Fig. 4(b)], it can be seen that the models predict quite different mean responses for pulse amplitudes that elicit fewer than 1000 spikes. There is a difference in the slopes of the functions and in the amount of variance. It is likely that behavioral threshold is reached with a smaller number of discharges, and hence some or all of a cochlear

\footnotetext{
${ }^{4}$ Unfortunately, signal detection theory cannot be used to explain the nature or behavior of UCL. It is possible that other neural mechanisms contribute to the sensation of UCL.
}

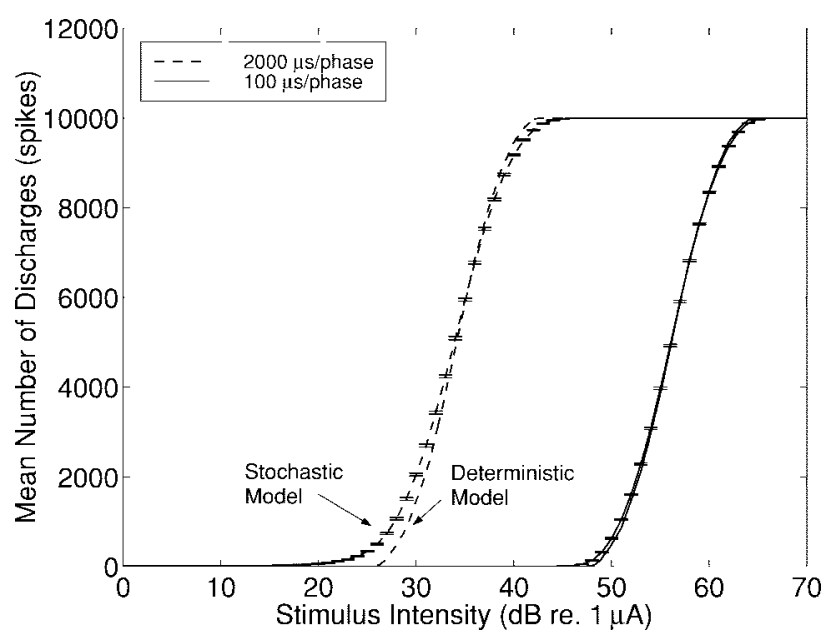

(a)

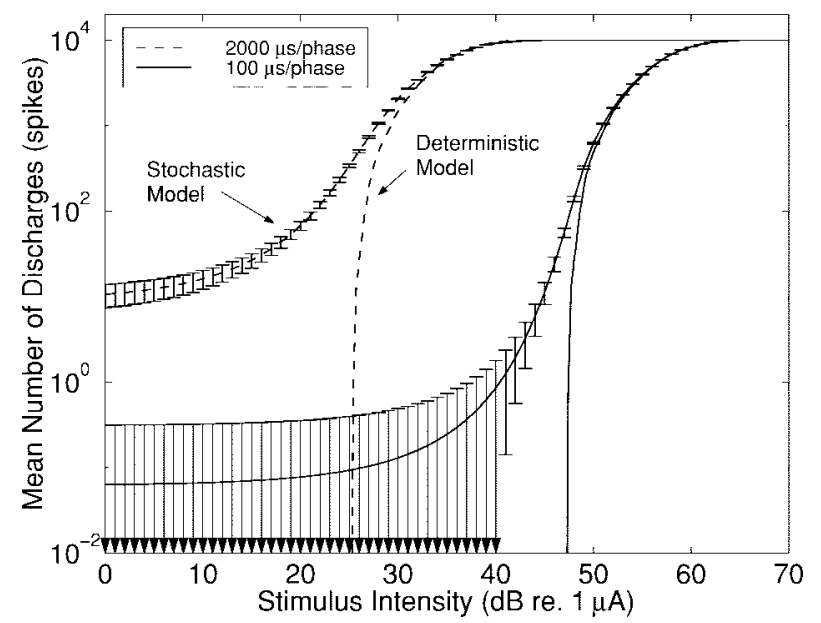

(b)

Fig. 4. Neural response versus stimulus intensity from a model of 10000 fibers in response to a single pulse of duration 100 and $2000 \mu$ s/phase (solid lines and dashed lines, respectively): (a) deterministic and stochastic model: linear vertical scale and (b) deterministic and stochastic model: logarithmic vertical scale.

implant's operating range may be in the region where the stochastic activity is significant. From this we expect that the deterministic and the stochastic models will have differing predictions of intensity perception as a function of phase duration and that these differences will be greater at lower stimulus intensities.

Neural response to a single pulse predicted for MP (solid line) and BP (dashed line) stimulation is plotted in Fig. 5. For the deterministic model, the excitation of fibers (shown by the slope of the curve) is faster for a MP stimulus than for a BP stimulus, because of the comparatively wider current spread (see [1, Fig. 9]). However, for the stochastic model, this effect is limited to high stimulus intensities and is less pronounced. In the region below $50 \mathrm{~dB}$ the growth of the response is dominated by the slopes of the fibers' I/O functions, not the current spread function. Again, a significant proportion of a cochlear implant's operating range may lie within the region below $50 \mathrm{~dB}$, where the mean responses of the two models diverge and where the variance is significant in the stochastic model. Similar to our hypothesis of the importance of pulse width, we 


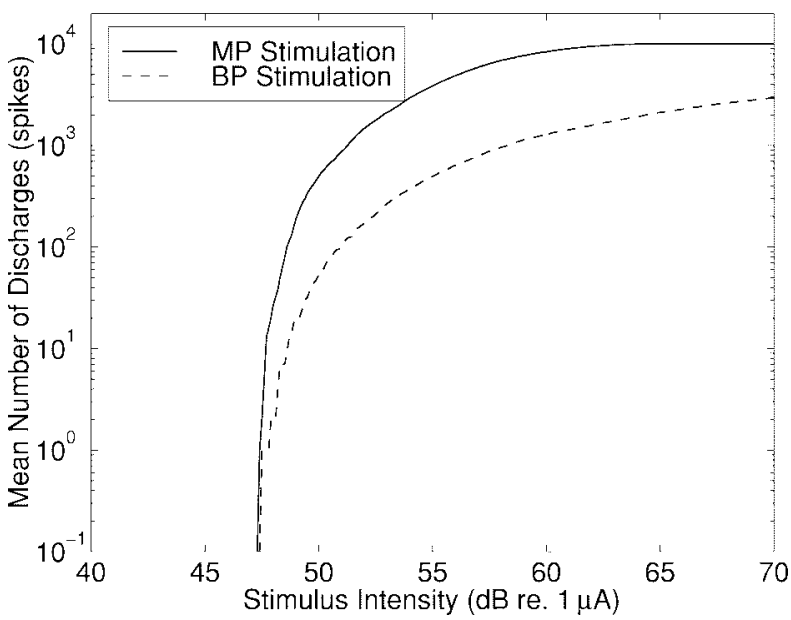

(a)

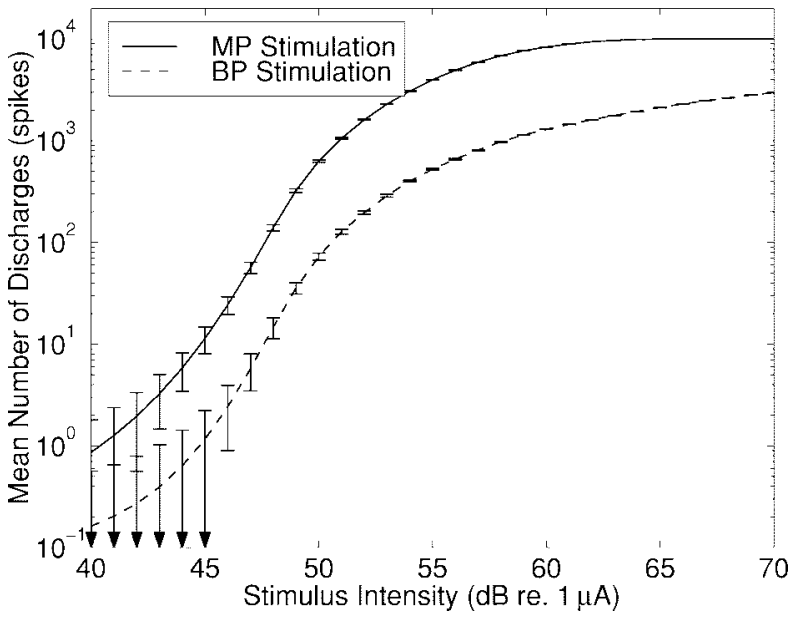

(b)

Fig. 5. Neural response versus stimulus intensity from a model of 10000 fibers in response to a single pulse for MP (solid line) and BP (dashed line) stimulation: (a) deterministic model and (b) stochastic model.

expect that BP and MP stimulation will also produce differing predictions in the stochastic and deterministic models and that these differences will be greater at lower stimulus intensities.

Furthermore, because of the difference in the response growth curves of the deterministic and stochastic models: 1) predictions of perception as a function of the number of pulses will differ for the two models and 2) predictions of IDL will differ.

We now look directly at how our model's predictions of intensity perception compare with psychophysical data.

\section{B. Threshold and Uncomfortable Loudness Versus Phase Duration: Dynamic Range}

Behavioral threshold and UCL versus phase duration data from two human subjects are plotted in Fig. 6. Using a BP electrode configuration, the stimuli for one of the subjects were single-cycle sinusoids, ${ }^{5}$ BP stimulation (from [29, Fig. 9]). The stimuli for the other subject were biphasic pulse trains delivered at 100 pps (from [37, Fig. 1]). The threshold versus

\footnotetext{
${ }^{5}$ For a single-cycle of sinusoidal current we define phase duration as half the period of the sinusoid.
}

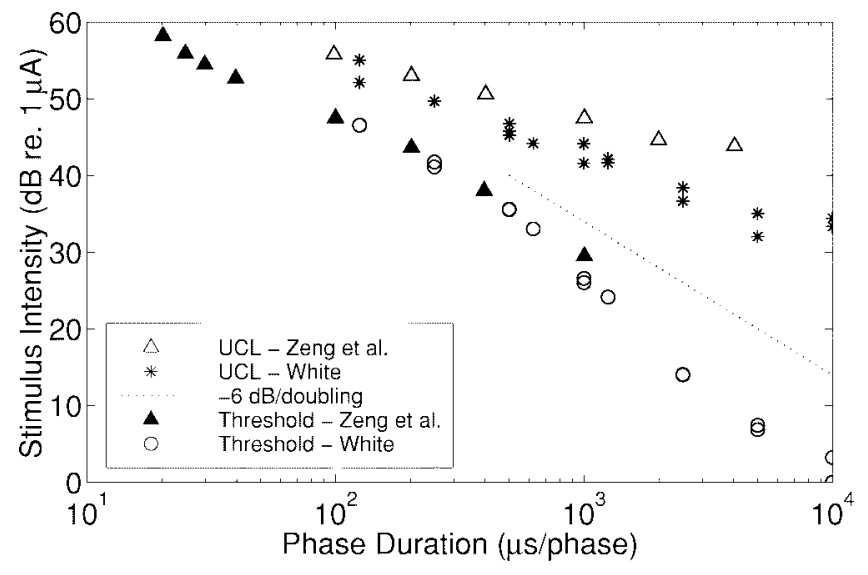

Fig. 6. Psychophysical data: Threshold and uncomfortable loudness from two human subjects (one from [37, Fig. 1] and another from [29, Fig. 9]) as indicted in the figure legend. Note that repeated measures for the one subject were taken at some phase durations as indicted.

phase duration curves have slopes that begin to steepen with phase durations greater than $500 \mu \mathrm{s} / \mathrm{phase}$ and the slope of the White data is steeper than $-6 \mathrm{~dB} /$ doubling in the region from 1000 to $2000 \mu \mathrm{s} / \mathrm{phase}$. These results are consistent with the data of Pfingst et al. collected in monkeys (see [15, Fig. 2]). In contrast, the UCL curves monotonically decrease in slope with increasing phase duration, causing the dynamic range to increase with increasing phase duration. This is consistent with other data from human subjects in response to pulsatile stimuli, e.g., see single-pulse data for phase durations between 200 and $10000 \mu \mathrm{s} /$ phase from one subject in [29, Fig. 7], averages across four subjects in [37, Table I], and averages across 14 subjects for low-rate pulse trains of phase durations between 24 and $408 \mu \mathrm{s} /$ phase in [38, Table IV].

Our model's prediction of these data are plotted in Fig. 7. The deterministic model predicts that the threshold and UCL curves will have identical slopes at each phase duration [Fig. 7(a)], such that the behavioral dynamic range will not change with phase duration. In contrast, the stochastic model accurately predicts the changes in slopes of the threshold and UCL curves at each phase duration [Fig. 7(b)] and the corresponding increase in dynamic range with increasing phase duration. It appears that UCL for these data corresponds to an $N_{u c l}$ for our model in the region of 100 to 1000 spikes.

Note that in Fig. 7(b) the model prediction of UCL for $N_{u c l}=100$ spikes begins to converge with its prediction of threshold. We believe [1] that this arises from the inaccuracy of the stochastic AN model at very low stimulus intensities for long pulses. Specifically, we believe that discharge probabilities at very low stimulus intensities for long pulses are actually considerably lower than those predicted by the model integrated-Gaussian function. See [1] for a discussion. More physiological data are required to increase the accuracy of the model in this region where the data are likely to deviate significantly from an integrated-Gaussian function.

\section{Effect of Electrode Configuration on Dynamic Range}

We present here behavioral dynamic range data from human subjects for different electrode configurations. Battmer et al. 


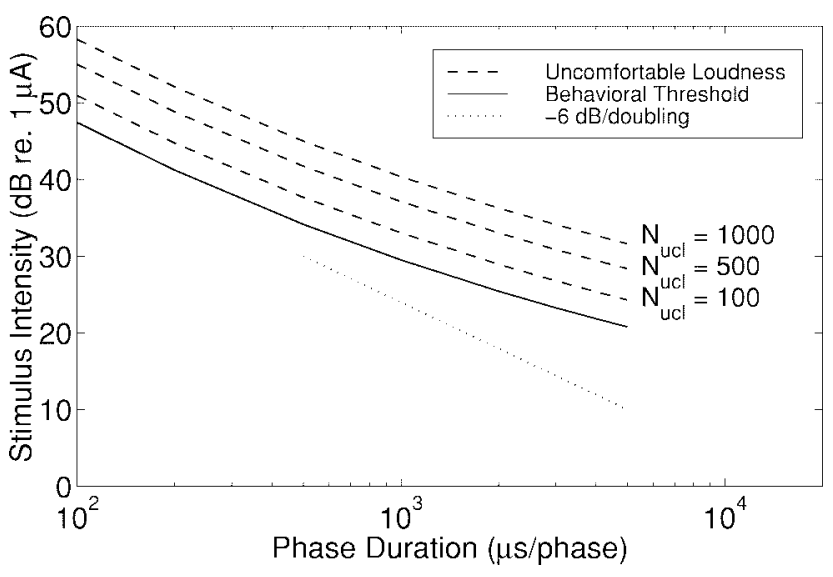

(a)

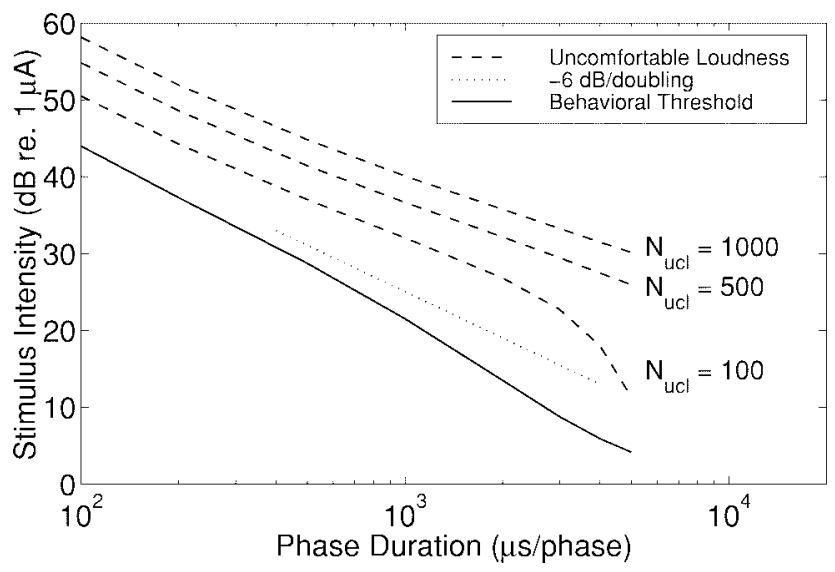

(b)

Fig. 7. Model predictions of threshold and uncomfortable loudness versus phase duration: (a) deterministic model and (b) stochastic model.

[39] have measured behavioral dynamic ranges from five subjects in response to pulses of widths $25,50,75,100,200$, and $400 \mu \mathrm{s} / \mathrm{ph}$ ase for BP and MP electrode configurations on two different electrodes.

These data are plotted in Fig. 8, along with model predictions of these data, as BP dynamic range divided by MP dynamic range. All of these data have a ratio of around one or less. The deterministic model predicts ratios between 2.7-3.9. In contrast the stochastic model predicts ratios of 0.6-1.2, depending on the value of $N_{u c l}$, which are much closer to the ratios observed in the psychophysical data. Like the dynamic range versus pulse width predictions of Section III-B, it appears that UCL for these data corresponds to an $N_{u c l}$ for our model in the region of 100 to 1000 spikes.

These data and model predictions are consistent with the data and model predictions shown in [33, Fig. 6-9]. Zwolan et al. (see [40, Table III]) have also found: 1) dynamic ranges in six human subjects to be on average approximately equal for BP and MP stimulation-in agreement with the Battmer et al. data plotted in Fig. 8 and 2) some subjects with smaller $\mathrm{BP}$ dynamic ranges than $\mathrm{MP}$-in agreement with the data plotted in [33, Fig. 6-9]. Similar BP and MP dynamic ranges have also been observed for stimuli very different from those presented and modeled here. Shannon has measured behavioral dynamic ranges in response to a $1000-\mathrm{Hz}, 300-\mathrm{ms}$ sinusoidal

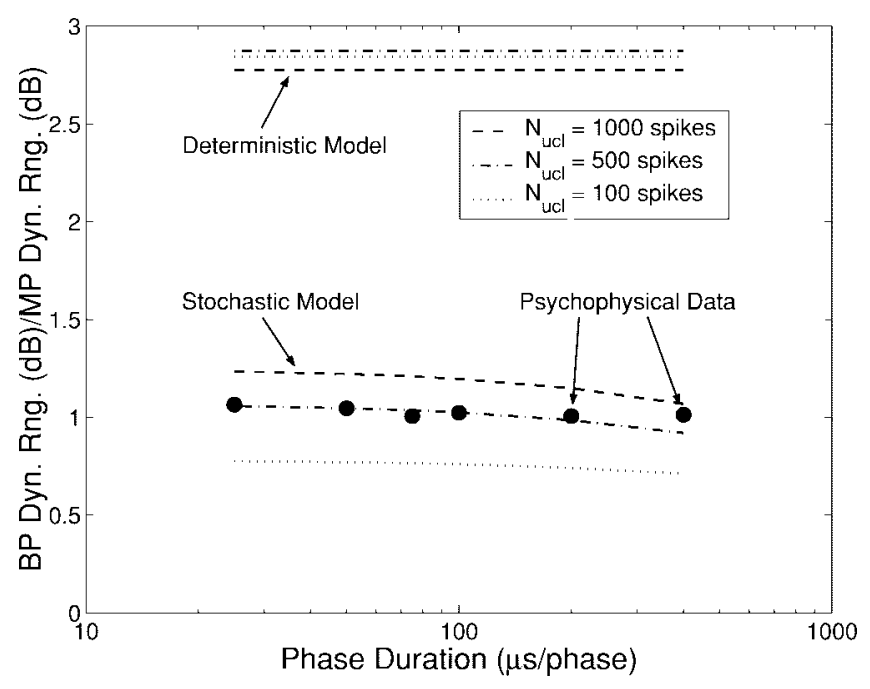

Fig. 8. BP dynamic range divided by MP dynamic range: psychophysical data (averages across five human subjects and two electrodes) from [39] and model predictions, for phase durations of $25,50,75,100,200$, and 400 $\mu \mathrm{s} / \mathrm{phase}$.

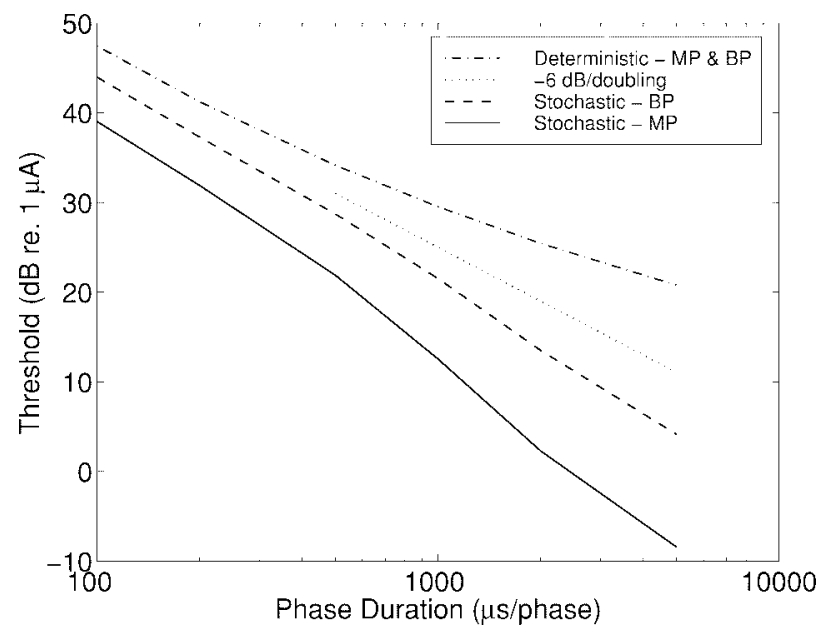

Fig. 9. Deterministic and stochastic model predictions of threshold versus phase duration (pulse width) for BP and MP stimulation.

stimulus [41] for a number of electrodes in a multielectrode array, using both BP and MP electrode configurations. Over the entire electrode array the dynamic ranges for $\mathrm{BP}$ and $\mathrm{MP}$ stimulation were approximately equal.

The model predictions can be better understood when the factors contributing to the growth in the total AN response are considered [see Fig. 5(a) and (b)]. In the deterministic model, thresholds do not change with electrode configuration [see Fig. 9], so only the effect of electrode configuration on UCL will alter the dynamic range. Only two factors contribute to excitation of fibers in the deterministic model: 1) the spread of current to fibers distant from the electrode and 2) the distribution single-fiber thresholds at any one location. If the former were the only factor (i.e., single-fiber thresholds in the deterministic model were all identical), then the BP:MP dynamic range ratio would be equal to the ratio of the current attenuation rates for the two modes $(=8)$. Indeed, for BP stimulation the first factor (" 1 ") is dominant: the spread of 
current is very narrow and, therefore, all the fibers in the vicinity of the electrode are excited at fairly low intensities, such that the excitation of fibers successively further away becomes the primary factor at intensities approaching UCL. In contrast, MP stimulation produces a wide spread of current, such that the second factor ("2") has a stronger impact at all intensities. The effect of the second factor, therefore, is to moderate the differences between the two modes of stimulation somewhat, more than halving the predicted BP:MP dynamic range ratio. ${ }^{6}$

However, this ratio is still approximately two to seven times greater than the ratios seen in the psychophysical data. The stochastic model accounts for this with two additional factors: 1) the distribution of single-fiber I/O function slopes and 2) the consequent difference in behavioral thresholds for BP and MP electrode configurations (see Fig. 9 for model predictions, [42, Fig. 6], and [43, Fig. 4(a)] for supporting psychophysical data). The former means that fibers with the lowest thresholds and the shallowest slopes will be excited at lower intensities than those with steeper slopes. In MP mode the wide spread of current "makes available" a greater pool of these sensitive, shallow-slope fibers. As a consequence, only a very small stimulus amplitude is required to excite these fibers sufficiently to generate a cochlear response sufficient for behavioral threshold. BP mode will excite a smaller number of these shallow-slope fibers. Therefore, a considerably higher amplitude is required to generate the same total cochlear response. At higher stimulus intensities near UCL, steep-slope fibers are responsible for almost all the changes in cochlear activity. Because only small changes in stimulus amplitude create large changes in neural activity, the difference in stimulus amplitudes required to elicit UCL for the two electrode types is generally ${ }^{7}$ not as great as that at behavioral threshold. Thus the stochastic model generally predicts BP : MP dynamic range ratios that are approximately one or less.

\section{Further Results}

A number of further predictions of psychophysical data, not shown in this paper due to space restrictions, have been obtained with this model [33]. Preprints of a manuscript in preparation that contains these results are available (download from http://www.bme.jhu.edu/ ibruce/papers/predict.htm or email: ibruce@bme.jhu.edu). In summary, these results are as follows.

1) Threshold Versus Number of Pulses (Temporal Integration): Both the psychophysical data from one human subject shown in [33, Fig. 6-12] and the data from five human subjects shown in [44, Fig. 5] indicate the steeper slopes of threshold versus number of pulses (temporal integration) curves for longer phase durations.

\footnotetext{
${ }^{6}$ If the range of thresholds (at a given cochlear place) is found through neurophysiological measurements to be more Gaussian than uniform as we assumed in our model, then the deterministic model's prediction of the BP : MP dynamic range ratio would further approach the behavioral data.

${ }^{7}$ If quite high stimulus levels are required to reach UCL (e.g., $N_{u c l}$ is quite large, or the total number fibers is small), the stochastic model will behave similarly to the deterministic model. In such cases, the difference in UCL levels for the two electrode types can be relatively large. As a consequence, BP : MP dynamic range ratios can become somewhat larger than one.
}

The stochastic model is able to predict this increase in slope with phase duration, whereas the deterministic model predicts zero slope for all phase durations.

2) Intensity Difference Limen: Both the deterministic and stochastic models agree with intensity difference limen data from [45] in that they predict an $\sim 8-\mathrm{dB}$ reduction in the maximum Weber fractions measured over the dynamic range of a subject. However, the deterministic model predicts very erratic Weber functions, with the predicted Weber fractions all underestimating the data, while the stochastic model predicts smooth Weber functions as seen in the data and Weber fractions in the same range as those of the data.

3) Effect of Number of Fibers on Threshold, Uncomfortable Loudness and Intensity Difference Limen: While both the deterministic and the stochastic model predict changes in dynamic range and Weber fraction with respect to threshold as the number of fibers is varied: 1) the deterministic model predicts practically no change in threshold with the number of fibers and 2) the deterministic model predicts decreases in dynamic range and Weber fraction with increasing neural survival, whereas the stochastic model predicts the opposite. Comparison of the model predictions with psychophysical data from a number of human subjects shown in [45, Fig. 7], whom we could expect to vary in the number of surviving fibers, suggests that the stochastic model, in contrast to the deterministic model, may account for some of the intersubject variability in the psychophysical data.

\section{DISCUSSION}

\section{A. Using the Model to Understand Psychophysical Behavior}

The relationship between cochlear response and behavior is defined by the central component of our model. We use a spike-counting model (i.e., perfect spatiotemporal summation) used in some models of normal hearing. This model is relatively simple to use. For example: 1) by adjusting the amplitude of a stimulus until the elicited cochlear output is equal to that elicited by another stimulus, the two stimuli can be made to evoke the same perceived intensity and 2) the output is a single mean and a single variance for each stimulus, so relatively simple statistical methods can be used to measure detectability or discriminability of stimuli.

Understanding how the cochlear response is affected by stimulus parameters, electrode configuration, nerve pathology, and nerve survival is necessary for understanding how these variables control behavioral response. The cochlear response is controlled by three primary factors: 1) The individual fiber I/O functions-we have shown that model predictions for a stepfunction (deterministic model) and for an integrated-Gaussian function (stochastic model) are very different. ${ }^{8}$ 2) Differences in fibers-the distribution of fiber I/O slopes is broad and

\footnotetext{
${ }^{8}$ Because AN fibers may only discharge in response to a fraction of the pulses in the stimulus, discharge probabilities for individual fibers are best visualized using a two to three decade logarithmic scale. Cochlear response functions (e.g., Fig. 4) should be viewed over a much larger range (e.g., six decades)!
} 
unimodal (and thresholds also vary among fibers). The deterministic model cannot incorporate the slopes of I/O functions, let alone their distribution. In contrast, the stochastic model is able to describe the distribution of slopes, such that fibers with shallow slopes contribute the majority of cochlear activity at low stimulus intensities. In sharp contrast, fibers with steep slopes contribute the majority of increase in cochlear activity at high stimulus intensities. 3) The large impact of phase duration (pulse width) on a fiber's discharge probability function.

The impact of the three primary factors, plus other factors such as the number of pulses, electrode configuration and nerve survival, can be understood and predicted. For example, in order to maintain the same level of audibility when the number of stimulus pulses is halved, pulse amplitude must be increased so that the average discharge probability is doubled. In a similar manner, in order to maintain the same perceived intensity when switching from MP to BP stimulation, pulse amplitude must be increased to compensate exactly for the reduction in discharge probability across the cochlea. Similarly, a cochlea with poor nerve survival requires a compensatory increase in pulse amplitude. Furthermore, the relative noise level of fibers may be quite different for different cochlear pathologies [46]. This directly impacts the slope of individual fibers and, therefore, the slope of total cochlear response. For example, in subjects with a pathology that causes fibers to be relatively noisy, one would expect dynamic ranges to be larger and intensity discrimination to be poorer, particularly at low intensities.

\section{B. Model Extensions}

In this paper, we have derived a model of intensity perception in cochlear implants users based on physiological data and have used this model to investigate a number of different psychophysical phenomena. In all the cases examined in this paper, the model predicts the perceptual performance of cochlear implant users significantly better when stochastic activity is included. However, extensions or revisions of this AN model may further improve predictions and our understanding of the functional significance of the physiology.

As we raised in [1], the neural section of our model is derived from physiological data collected in cats. Further physiological data may be collected from humans using cochlear implant telemetry and noninvasive electrophysiology that should prove useful in refining our simple model of current spread and neural response. A model of current spread in the human cochlea constructed from human cochlear sections [47] may also help to this end.

Another extension to the model would be to allow for other sources of noise. For instance, the survival of inner hair cells in some subjects could result in some remaining synapse-driven spontaneous activity in the AN. This would affect the amount of noise present in the total AN response. Other sources of noise may also be present in more central sections of the auditory pathways. The effects of both of these potential noise sources can be included in our psychophysical model if their behavior is known. We have conducted initial investigations into such effects, where we have assumed that the additional noise is unaffected by stimulus parameters. One consequence of this noise is that thresholds increase with increasing noise levels such that the stochastic model behavior tends toward that of the deterministic model. This may account for those strength-duration curves in [15, Fig. 2] that are higher and flatter. Central noise may also account for the relatively high Weber fractions measured in some subjects (see [45, Fig. 6]). Furthermore, from preliminary simulation results it appears that additional noise may improve the predictions of the stochastic model more than the predictions of the deterministic model.

In summary, the results listed in the preceding paragraph indicate that such additional noise sources may improve predictions to some small extent and may be useful in understanding intersubject variability. However, such hypothesized noise sources have not been well characterized. This contrasts with stochastic activity occurring in auditory nerve fibers, for which there are good measurements, significant evidence of its source, and accurate predictive biophysical models. Furthermore, we have shown in this paper that inclusion of this single noise mechanism in a simple cochlear model enables accurate prediction of a wide-range of psychophysical behavior.

By changing parameters of the model to reduce the amount of stochastic activity we may also account for such data that lie somewhere between the deterministic model and the stochastic model predictions. For instance, particularly focused current fields or extremely low neural survival may cause higher probabilities of firing at stimulus intensities within the behavioral operating range. Because neural responses at high discharge probabilities exhibit relatively little variability, stochastic and deterministic model predictions are similar under such conditions.

The physiological data on which our model is based are from acutely-implanted, normal-hearing animals. This model does not take into account the effects that etiology, prolonged deafness and implantation have on the response of AN fibers to electrical stimulation [46]. An extension to this study could be to model the effects of various etiologies on single-fiber I/O functions and current spread.

Only responses to stimulation from a single electrode have been investigated in this paper. In order to model responses to stimulation from multiple electrodes, even at moderate pulse rates, refractory effects should be incorporated [2] when the electrodes are stimulating overlapping populations of fibers. Also, loudness summation effects may need to be considered when the neural populations excited do not overlap [30], [48], [49].

In this paper we have limited our investigation to low pulserate stimuli. With the pulse-train model developed in [2], we now have a good tool for extending this investigation to the prediction of psychophysical data for moderate stimulation rates (200-1000 pps). However, to improve this model for moderate and high pulse-rate (>1000 pps) stimulation, neurophysiological data must be collected over a range of discharge probabilities (possibly as low as 0.01 or lower) at such pulse rates. Preliminary physiological data [5], [50], [51] reveal interpulse interactions occurring at high pulse rates that 
can significantly increase or decrease the level of stochastic activity in a fiber. This leads us to believe that stochastic activity in the AN may play an even more important role in predicting and understanding behavioral responses to high pulse-rate stimuli.

\section{Applicability of the Model}

The results presented in this paper have important consequences for physiological studies, for investigation of neural sound coding and for speech processing strategies. For physiological studies, the results suggest that "discharge probability versus stimulus intensity" functions should be measured. Such measurements will provide a far more accurate description of spatio-temporal patterns of AN response and better enable us to understand how information could be coded. For psychophysical studies, our results indicate that many perceptual measures are better predicted by the stochastic model. The source of this improvement is in the description of the most peripheral section of the auditory pathways. It is, therefore, suggested that consideration of stochastic activity in $\mathrm{AN}$ responses may produce better predictions and explanations of a range of other psychophysical measures not investigated in this paper. Furthermore, it is likely that the "bare-bones" psychophysical section of this model should be extended to predict additional psychophysical measures with more accuracy.

We have investigated neural and psychophysical responses to stimuli with a wide range of phase durations. Higher rate pulsatile stimuli are typically used in modern cochlear implants, necessitating short phase durations. One might, therefore, wonder as to the relevance of studying very long pulse widths. There are two reasons: 1) We believe an important feature of our AN model is the slope and shape of the singlefibers' "probability versus stimulus intensity" functions. We have direct experimental control of this slope by changing phase duration. By so changing the fibers' slopes we have observed large, correlated changes in psychophysical measures. We consider fiber slope to be the dominant feature in much of the behavior investigated in this paper. Therefore, we believe the ability to manipulate this feature to be fundamental to developing an understanding of cochlear response to electrical stimulation. 2) There is a relatively large body of psychophysical data available in which long phase duration stimuli were used.

It might appear that a three-piece linear fit to single-fiber I/O data would be an adequate AN model, instead of an integratedGaussian fit. Quite to the contrary, simulations indicate that such models behave more like the deterministic model when used to predict behavioral threshold and threshold-dependent psychophysical measures (e.g., dynamic range, IDL as a function of loudness). While the three-piece function does produce a graded discharge probability over a small stimulusintensity range $(2-4 \mathrm{~dB})$, the discharge probability is zero or one over all the remaining intensity range. This is similar to the deterministic model, in which discharge probability is zero or one for all stimulus intensities. The results of this paper indicate that behavioral threshold is particularly sensitive to the low-probability "tail" of single-fiber I/O functions, and consequently predictions of behavioral threshold (and related measures) using the three-piece model are only slightly better than those of the deterministic model. This can be most clearly understood by plotting all three functions on a log-probability, log-amplitude $(\mathrm{dB})$ scale. The three-piece function and the deterministic model appear very similar to each other when contrasted to the integrated-Gaussian function.

As we raised in [1], the concepts considered in this paper are already being applied to the design of cochlear implants. These concepts have direct relevance to both the: 1) effectiveness and 2) safety of cochlear implants. In particular, let us consider current high pulse-rate stimulation strategies. 1) If it were not for the inherently stochastic nature of the AN, stimulus pulse-rates higher than a fiber's maximum discharge-rate might severely distort the temporal representation of a speech signal. In contrast, the combined activity of many stochastic fibers, each firing at a low rate, should accurately represent the temporal features of a speech signal. 2) Nerve damage studies have found that stimulation at high pulse-rates and high amplitudes does induce serious injury to the nerve [52], [53]. However, at lower stimulus amplitudes that are within the animal's normal loudness range, nerve damage is not observed [54]. A likely explanation for these results is directly related to the stochastic nature of $\mathrm{AN}$ responses. If we stimulate the nerve with a high pulse-rate, low-amplitude stimulus we would expect that each fiber's discharge probability per pulse would be low, i.e., the fiber's discharge rate would be much lower than the stimulus's pulse rate [2], [55]. As a consequence, no damage to the fibers would occur. However, if the stimulus was high in amplitude, fiber discharge probabilities per pulse would become high, i.e., fiber discharge rates would indeed become too high [2], [55] and cause injury to the nerve.

In $[33$, ch. 8], we have analyzed the stochastic model output to determine what discharge probabilities per pulse are reached on individual fibers in "extreme cases." With the phrase "extreme cases" we refer to stimulus (and neural) parameters that are likely to produce high discharge probabilities, but which are also likely to be within normal operating levels of cochlear implant users. From this analysis, even in the most extreme cases with a BP electrode configuration, individual spike probabilities are low except on a very few fibers. This means that all but these few fibers will be responding at a fraction of the pulse rate. Furthermore, we hypothesize that discharge probabilities for cochlear implant users are very likely to be significantly lower than for our extreme cases. First, most speech processing strategies will stimulate using multiple electrodes, such that the excitation contributing to the loudness of the stimulus will be distributed amongst more fibers than when using a single electrode. Thus, discharge probabilities on individual fibers will be lower for multielectrode stimulation. Second, pulse rates of 200-800 pps and higher are being used in implants. Increasing the pulse rate will cause more pulses to fall within the temporal integrator window. Therefore, UCL will be reached at even lower individual discharge probabilities. Third, MP stimulation is commonly used in cochlear implants. The results in [33, Table VIII.1] indicate that MP stimulation will result in quite low fiber discharge probabilities. Fourth, these results are for a model cochlea 
of 10000 fibers, which is a conservative estimate of nerve survival in implant users. From [34], nerve survival may be higher in the majority of users. Greater survival will also lead to a greater distribution of excitation amongst the fibers, again leading to reduced discharge probabilities on individual fibers. Finally, although this is more speculative, preliminary data from Dynes [5] indicate that high pulse-rate stimulation may further increase the noise level of AN fibers. Dynes found that a subthreshold pulse increased the relative noise level for subsequent pulses, and for a pulse train that produces low discharge probabilities (as we postulate is the case for almost all fibers) the majority of pulses in the train will be subthreshold. These preliminary physiological data are consistent with some psychophysical data for high-rate stimulation [29]. Since many implants now use high stimulus pulse-rates, fiber noise-levels may indeed be higher than those used in the model. Model simulations using higher fiber noiselevels indicate that there is a greater distribution of excitation amongst the fibers (i.e., a greater "spread" of excitation), again leading to reduced discharge probabilities per fiber.

\section{ACKNOWLEDGMENT}

The authors would like to thank L. Cohen, P. Blamey, and the four anonymous reviewers for invaluable comments on the manuscript.

\section{REFERENCES}

[1] I. C. Bruce, M. W. White, L. S. Irlicht, S. J. O’Leary, S. Dynes, E. Javel, and G. M. Clark, "A stochastic model of the electrically stimulated auditory nerve: Single-pulse response," IEEE Trans. Biomed. Eng., vol. 46, pp. 617-629, June 1999.

[2] I. C. Bruce, L. S. Irlicht, M. W. White, S. J. O'Leary, S. Dynes, E. Javel, and G. M. Clark, "A stochastic model of the electrically stimulated auditory nerve: Pulse-train response," IEEE Trans. Biomed. Eng., vol. 46, pp. 630-637, June 1999.

[3] S. J. O'Leary, L. S. Irlicht, I. C. Bruce, M. W. White, and G. M. Clark, "Variance of spike rate from auditory nerve following electrical pulsetrain stimulation of cochlea: An experimental and modeling study," submitted for publication.

[4] L. S. Irlicht and G. M. Clark, "Control strategies for neurons modeled by self-exciting point processes," J. Acoust. Soc. Amer., vol. 100, no. 5, pp. 3237-3247, 1996.

[5] S. Dynes, "Discharge characteristics of auditory nerve fibers for pulsatile electrical stimuli," Ph.D. dissertation, Massachusetts Inst. Technol., Cambridge, MA, 1996.

[6] J. T. Rubinstein, "Threshold fluctuations in an $\mathrm{N}$ sodium channel model of the node of Ranvier," Biophysical J., vol. 68, pp. 779-785, 1995.

[7] M. W. White, C. C. Finley, and B. S. Wilson, "Electrical stimulation model of the auditory nerve: Stochastic response characteristics," in Proc. 9th Аnnu. Conf. IEEE, Engineering in Medicine and Biology Society, Boston, MA., 1987, pp. 1906-1907.

[8] I. J. Hochmair-Desoyer, E. S. Hochmair, H. Motz, and F. Rattay, "A model for the electrostimulation of the nervus acusticus," Neuroscience, vol. 13 , no. 2, pp. 553-562, 1984

[9] M. W. White, "Design considerations of a prosthesis for the profoundly deaf," Ph.D. dissertation, Univ. California, Berkeley, 1978.

[10] W. F. Sewell, "Neurotransmitters and synaptic transmission," in The Cochlea, P. Dallos, A. N. Popper, and, R. R. Fay, Eds. SpringerVerlag, New York: 1996, ch. 9, pp. 503-533.

[11] N. Y. S. Kiang, E. C. Moxon, and R. A. Levine, "Auditory-nerve activity in cats with normal and abnormal cochleas," in Sensorineural Hearing Loss, G. E. W. Wolstenholme and J. Knight, Eds. London, U.K.: Churchill, 1970, p. 241

[12] A. A. Verveen and H. E. Derksen, "Fluctuation phenomena in nerve membrane," Proc. IEEE, vol. 56, pp. 906-916, June 1968.

[13] C. Van Den Honert and P. H. Stypulkowski, "Physiological properties of the electrically stimulated auditory nerve-II: Single fiber recordings," Hear. Res., vol. 14, pp. 225-243, 1984
[14] B. E. Pfingst, "Comparisons of psychophysical and neurophysiological studies of cochlear implants," Hear. Res., vol. 34, pp. 243-252, 1988.

[15] B. E. Pfingst, D. R. De Haan, and L. A. Holloway, "Stimulus features affecting psychophysical detection thresholds for electrical stimulation of the cochlea-I: Phase duration and stimulus duration," J. Acoust. Soc. Amer., vol. 90, no. 4, pp. 1857-1866, 1991

[16] B. E. Pfingst, "Psychophysical constraints on biophysical/neural models of threshold," in Cochlear Implants-Models of the Electrically Stimulated Ear, J. M. Miller and F. A. Spelman, Eds. NY: Springer-Verlag, 1990, pp. 161-185.

[17] R. V. Shannon, "A model of threshold for pulsatile electrical stimulation of cochlear implants," Hear. Res., vol. 40, pp. 197-204, 1989.

[18] S. J. O'Leary, G. M. Clark, and Y. C. Tong, "Model of discharge rate from auditory nerve fibers responding to electrical stimulation of the cochlea: Identification of cues for current and time-interval coding," Ann. Otol. Rhinol. Laryngol., vol. 104, no. 166, pp. 121-123, 1995.

[19] C. M. McKay and H. J. McDermott, "Loudness perception with pulsatile electrical stimulation: The effect of interpulse intervals," J. Acoust. Soc. Amer., vol. 104, no. 2, pp. 1061-1074, 1998.

[20] M. W. White, "Neurophysiological and psychophysical considerations in the design of a cochlear prosthesis," Ann. Otol. Rhinol. Laryngol., vol. 96 , no. 128 , p. $42,1987$.

[21] _ "The multichannel cochlear prosthesis: Channel interactions," in Proc. 6th Annu. Conf. IEEE Engineering in Medicine and Biology Society -Frontiers of Engineering and Computing in Health Care, Los Angeles, CA, 1984, pp. 396-400.

[22] D. M. Green and J. A. Swets, Signal Detection Theory and Psychophysics. New York: Wiley, 1969; reprinted: New York: Krieger, 1973.

[23] M. C. Teich and G. Lachs, "A neural-counting model incorporating refractoriness and spread of excitation-I. Application to intensity discrimination," J. Acoust. Soc. Amer., vol. 66, pp. 1738-49, 1979.

[24] N. I. Durlach, L. D. Braide, and Y. Ito, "Toward a model of discrimination of broadband signals," J. Acoust. Soc. Amer., vol. 80, no. 1, pp. 63-72, 1986.

[25] W. S. Hellman and R. P. Hellman, "Intensity discrimination as the driving force for loudness. Application to pure tones in quiet," J. Acoust. Soc. Amer., vol. 87, pp. 1255-65, 1990

[26] M. M. Merzenich and M. W. White, "Cochlear implants: The interface problem," in Functional Electrical Stimulation: Applications in Neural Prostheses, vol. 3, F. T. Hambrecht and J. B. Reswick, Eds. New York: Marcel Dekker, 1977, pp. 321-340.

[27] S. J. O'Leary, R. C. Black, and G. M. Clark, "Current distributions in the cat cochlea: A modeling and electrophysiological study," Hear Res., vol. 18, pp. 273-281, 1985.

[28] R. V. Shannon, "Psychophysics," in Cochlear Implants: Audiological Foundations, R. S. Tyler, Ed. San Diego, CA: Singular Publishing Group, 1993, pp. 357-388.

[29] M. W. White, "Psychophysical and neurophysiological considerations in the design of a cochlear prosthesis," Audiol. Ital., vol. 1, no. 2, pp. 77-117, 1984.

[30] M. W. White, M. M. Merzenich, and J. N. Gardi, "Multichannel cochlear implants: Channel interactions and processor design," Arch. Otolaryngol., vol. 110, pp. 493-501, 1984.

[31] C. Zhang and F.-G. Zeng, "Loudness of dynamic stimuli in acoustic and electric hearing," J. Acoust. Soc. Amer., vol. 102, no. 5, pt. 1, pp. 2925-2934, 1997.

[32] H. Levitt, "Transformed up-down methods in psychoacoustics," $J$. Acoust. Soc. Amer., vol. 49, no. 2, pp. 467-477, 1970.

[33] I. C. Bruce, "Spatiotemporal coding of sound in the auditory nerve for cochlear implants," Ph.D. dissertation, Univ. Melbourne, Melbourne, Australia, 1997.

[34] R. Hinojosa and M. Marion, "Histopathology of profound sensorineural deafness," Ann. N.Y. Acad. Sci., vol. 405, pp. 459-484, 1983.

[35] H. Fletcher and W. Munson, "Loudness, its definition, measurement, and calculation," J. Acoust. Soc. Amer., vol. 5, pp. 82-108, 1933.

[36] E. M. Relkin and J. R. Doucet, "Is loudness simply proportional to the auditory nerve spike count?," J. Acoust. Soc. Amer., vol. 101, no. 5, pt. 1, pp. 2735-2740, 1997.

[37] F.-G. Zeng, J. J. Galvin III, and C. Zhang, "Encoding loudness by electric stimulation of the auditory nerve," NeuroReport, vol. 9, pp 1845-1848, 1998.

[38] B. E. Pfingst, T. A. Zwolan, and L. A. Holloway, "Effects of stimulus configuration on psychophysical operating levels and on speech recognition with cochlear implants," Hear. Res., vol. 112, pp. 247-260, 1997.

[39] R.-D. Battmer, D. Gnadeberg, E. Von Wallenberg, E. Lehnhardt, and D. J. Allum, "A study of monopolar and bipolar stimulation modes with 
a modified nucleus mini-22 cochlear implant," Adv. Otorhinolaryngol., vol. 48, pp. 9-16, 1993.

[40] T. A. Zwolan, P. A. Kileny, C. Ashbaugh, and S. A. Telian, "Patient performance with the Cochlear Corporation ' $20+2$ ' implant: Bipolar versus monopolar activation," Amer. J. Otol., vol. 17, no. 5, pp. 717-723, 1996.

[41] R. V. Shannon, "Multichannel electrical stimulation of the auditory nerve in man-I: Basic psychophysics," Hear. Res., vol. 11, pp. 157-189, 1983

[42] C. A. Miller, K. E. Woodruff, and B. E. Pfingst, "Functional responses from guinea pigs with cochlear implants-I: Electrophysiological and psychophysical measures," Hear. Res., vol. 92, no. 1/2, pp. 85-99, 1995.

[43] D. W. Smith and C. C. Finley, "Effects of electrode configuration on psychophysical strength-duration functions for single biphasic electrical stimuli in cats," J. Acoust. Soc. Amer., vol. 102, no. 4, pp. 2228-2237, 1997.

[44] A. K. Moon, T. A. Zwolan, and B. E. Pfingst, "Effects of phase duration on detection of electrical stimulation of the human cochlea," Hear. Res., vol. 67, pp. 166-178, 1993.

[45] D. A. Nelson, J. L. Schmitz, G. S. Donaldson, N. F. Viemeister, and E. Javel, "Intensity discrimination as a function of stimulus level with electric stimulation," J. Acoust. Soc. Amer., vol. 100, no. 4, pp. 2393-2414, 1996.

[46] R. K. Shepherd and E. Javel, "Electrical stimulation of the auditory nerve-I: Correlation of physiological responses with cochlear status," Hear. Res., vol. 108, pp. 112-144, 1997.

[47] L. T. Cohen, E. Saunders, P. A. Busby, and G. M. Clark, "Place information in cochlear implants: An integrated approach employing psychophysics and modeling," in Program and Abstracts 1997 Conf. Implantable Auditory Prostheses. Pacific Grove, CA: Asilomar Conf. Ctr., 1997.

[48] C. M. McKay, H. J. McDermott, and G. M. Clark, "Loudness summation for two channels of stimulation in cochlear implants: Effects of spatial and temporal separation," Ann. Otol. Rhinol. Laryngol., vol. 104, no. 166, pp. 230-233, 1995.

[49] R. V. Shannon, "Multichannel electrical stimulation of the auditory nerve in man-II: Channel interaction," Hear. Res., vol. 12, pp. 1-16, 1983.

[50] J. T. Rubinstein, A. J. Matsuoka, P. J. Abbas, and C. A. Miller, "The neurophysiological effects of simulated auditory prosthesis stimulation," Dept. Otolaryngol.-Head and Neck Surg. and Dept. Speech Pathol., Audiol., Univ. Iowa, Iowa City, IA, 2nd Quarterly Progress Rep. NO1DC-6-2111, 1997.

[51] C. A. Miller, P. J. Abbas, J. T. Rubinstein, and A. J. Matsuoka, "The neurophysiological effects of simulated auditory prosthesis stimulation,"
Dept. Otolaryngol.- Head and Neck Surg. and Dept. Speech Pathol., Audiol., Univ. Iowa, Iowa City, IA, 3rd Quarterly Progress Rep. NO1DC-6-2111, 1997.

[52] M. Tykocinski, R. K. Shepherd, and G. M. Clark, "Reduction in excitability of the auditory nerve following electrical stimulation at high stimulus rates," Hear. Res., vol. 88, pp. 124-142, 1995.

[53] "Acute effects of high-rate stimulation on auditory nerve function in guinea pigs," Ann. Otol. Rhinol. Laryngol., vol. 104, no. 166, pp. 71-74, 1995.

[54] J. Xu, R. K. Shepherd, R. E. Millard, and G. M. Clark, "Chronic electrical stimulation of the auditory nerve at high stimulus rates: A physiological and histopathological study," Hear. Res., vol. 105, pp. $1-29,1997$.

[55] E. Javel, Y. C. Tong, R. K. Shepherd, and G. M. Clark, "Responses of cat auditory nerve fibers to biphasic electrical current pulses," Ann. Otol. Rhinol. Laryngol., vol. 96, no. 128, pp. 26-30, 1987.

Ian C. Bruce (S'96-M'98), for a photograph and biography, see p. 628 of the June 1999 issue of this Transactions.

Mark W. White (M'79), for a photograph and biography, see p. 628 of the June 1999 issue of this TransaCtions.

Laurence S. Irlicht (M'94), for a photograph and biography, see p. 628 of the June 1999 issue of this Transactions.

Stephen J. O'Leary, for a photograph and biography, see p. 628 of the June 1999 issue of this Transactions.

Graeme M. Clark, for a photograph and biography, see p. 629 of the June 1999 issue of this TRANSACTIONS. 


\section{University Library}

\section{- M M N E R VA A gateway to Melbourne's research publications}

Minerva Access is the Institutional Repository of The University of Melbourne

Author/s:

Bruce, Ian C.;White, Mark W.;Irlicht, Laurence S.;O'Leary, Stephen J.;Clark, Graeme M.

Title:

The effects of stochastic neural activity in a model predicting intensity perception with cochlear implants: low-rate stimulation

Date:

1999

\section{Citation:}

Bruce, I. C., White, M. W., Irlicht, L. S., O'Leary, S. J., \& Clark, G. M. (1999). The effects of stochastic neural activity in a model predicting intensity perception with cochlear implants: low-rate stimulation. IEEE Transactions on Biomedical Engineering, December, 46(12), 1393-1404.

Persistent Link:

http://hdl.handle.net/11343/27532 